\title{
Treatment Results of Intralesional Steroid Injection and Topical Steroid Administration in Pregnant Women with Idiopathic Granulomatous Mastitis
}

\author{
(D) Osman Toktas ${ }^{1}$, (D) Nurşen Toprak ${ }^{2}$ \\ ${ }^{1}$ Department of Surgery, Van Yüzüncü Yıl University Faculty of Medicine, Van, Turkey \\ ${ }^{2}$ Department of Radiology, Van Yüzüncü Yıl University Faculty of Medicine, Van, Turkey
}

\begin{abstract}
Objective: Idiopathic granulomatous mastitis (IGM) is an inflammatory and chronic benign breast disease that has proven difficult to diagnose and treat. Since most treatment modalities cannot be used in pregnant patients, the choice of treatment is more difficult and the need for surgery is more pressing. In this first and innovative study, we assess the results of local corticosteroid therapy of IGM in pregnant women.

Materials and Methods: Pregnant women with IGM were evaluated between June 2017 and May 2019. The six pregnant women were treated using intralesional steroid injections and topical steroid administration. The treatment response was evaluated, both clinically and radiologically, at the end of 2 weeks and once more at the end of 1 month.

Results: The median patient age was 26 years. The mean duration of complaints was 4.3 months. The median number of children was 2 , and the mean breastfeeding time was 41 months. The predominant complaints at onset were a breast mass or local pain and inflammation in four (66.7\%) patients and a breast mass with pain and without signs of local skin inflammation in two (33.3\%) patients. Diagnosis was made using a tru-cut biopsy in two patients, and with an incisional biopsy in four patients who had abscess drainage and fistulation to the skin. Five (83.3\%) patients achieved a complete response, and one (16.7\%) patient responded only partially after the first course of treatment. A second course of treatment was given to the patient with partial response. All patients achieved complete response at the end of the second course of treatment. The mean follow-up time was 19.5 months. During the follow-up period, one patient experienced a recurrence at 4 months after giving birth, and she then received a third course of treatment. Topical and systemic side effects of the corticosteroids were not observed in any patient.
\end{abstract}

Conclusion: While the state of pregnancy generally precludes the use of most drugs, the use of local corticosteroid in the treatment of IGM is effective in terms of treatment response, treatment duration, need for surgery, and reduced recurrence and side effects.

Keywords: Idiopathic granulomatous mastitis, pregnant women, steroid injection, topical steroid

Cite this article as: Toktaş O, Toprak N. Treatment Results of Intralesional Steroid Injection and Topical Steroid Administration in Pregnant Women with Idiopathic Granulomatous Mastitis. Eur J Breast Health 2021; 17(3): 283-287

\section{Key Points}

- There is no consensus on the optimal treatment of IGM since its etiology is presently unknown due to insufficient case series and lack of prospective studies.

- Idiopathic granulomatous mastitis (IGM) is an inflammatory and chronic benign disease of the breast, which has long proven difficult to diagnose and treat. Since most treatment modalities cannot be used in pregnant patients, choice of treatment is more difficult.

- Pregnant women who were diagnosed with IGM and received treatment with local corticosteroids with at least 12 months of follow-up were included in the study. For patients treated with local steroids, protocol included an intralesional $40 \mathrm{mg}$ methylprednisolone injection into the breast skin affected by mastitis and administration of topical $0.125 \%$ prednisolone twice a day, on alternate days, for 4 weeks.

- While pregnancy argues against the use of many drugs, the use of local corticosteroid in the treatment of IGM is effective in terms of response to treatment, duration of treatment, need for surgery, and reduced recurrence and side effects. 


\section{Introduction}

Idiopathic granulomatous mastitis (IGM) is a benign inflammatory disorder and rare cause of chronic mastitis. There is no consensus on the optimal treatment of IGM, since its etiology is presently unknown given insufficient case series and lack of prospective studies (1). Antibiotic, corticosteroid, immunosuppressant, methotrexate, colchicine, and surgical treatment modalities have been previously used in IGM treatment. However, at present, since many medical treatments cannot be used in pregnant patients with IGM, corticosteroid and surgery are the most commonly used treatment modalities (2-4). Surgery carries with it cosmetic and healing problems; by contrast, long-term and high-dose use of corticosteroid has side effects $(5,6)$. Some recent studies have used steroids locally. These studies have shown that local corticosteroid was effective, better tolerated, and caused fewer side effects. In addition, studies have shown that steroid therapy results in faster recovery than expectant management and other systemic treatment $(1,4,5)$. In this study, intralesional steroid injections with concomitant topical steroids are assessed in the treatment of IGM in pregnant women.

\section{Materials and Methods}

Data collected between June 2017 and May 2019 were analyzed retrospectively. Pregnant patients who visited the breast clinic with complaints of a breast mass, breast pain, and/or local erythema were evaluated clinically and radiologically. Patients with suspected IGM underwent core needle and incisional biopsy depending on their clinical presentation. The diagnosis of IGM was made histopathologically. In the histopathological diagnosis of each patient, infectious granulomatous mastitis agents were, excluded based on the results of Gram, periodic-acid-Schiff, EhrlichZiehl-Neelsen, and Giemsa staining. Pregnant women who were diagnosed with IGM and received local corticosteroid treatment with at least 12 months of follow-up were included in the study. For patients treated with local steroids, the protocol included intralesional injection of $40 \mathrm{mg}$ methylprednisolone (Prednol-L 40 mg ampule; Mustafa Nevzat Pharmaceuticals, İstanbul, Turkey) into the breast skin affected by mastitis and topical administration of $0.125 \%$ prednisolone (Prednol-a30 gr pomade; Mustafa Nevzat Pharmaceuticals, İstanbul, Turkey) twice a day, on alternate days, for 4 weeks. Patients who have diffuse disease, by which lesions cover more than two quadrants of the breast, received injection at different points at $5 \mathrm{~cm}$ intervals. In patients with multiple lesions, if lesions were more than $5 \mathrm{~cm}$ apart, lesions were injected separately, and if the lesions were less than $5 \mathrm{~cm}$ apart, the larger one was injected.

Patients with recurrent disease, patients for whom use of corticosteroids was contraindicated, and patients aged $<18$ years were excluded from the study. Patients were followed up to assess treatment results. Treatment responses were evaluated both clinically and radiologically after 2 weeks and again after 1 month. The responses were categorized as "complete response," "partial response," "no response," or "worsening disease." Patients with "complete response" were assigned follow-up, while patients with "partial response," "no response," or "worsening disease" received the second and, if necessary, third courses of treatment with monthly check-ups until the disease completely resolved (Figures 1a and b). Surgical treatment was a second option for all patients who
This study was approved by the Local Ethics Committee of Yüzüncü Y1l University, Van, Turkey, with the registration number of 2020/0433. Informed consent was received from each patient.

\section{Results}

Six women in their third trimester of pregnancy were included in the study. The disease localized bilaterally in one patient, in the left breast in three patients, and in the right breast in two patients. The mean patient age was 26 (range: 23-33) years. None of the patients had comorbid medical condition, but one patient had a history of smoking. The mean duration of complaints was 4.3 months. While the mean number of children was 2 (range: $2-5$ ), the mean breastfeeding time was 41 (range, 24-72) months. Predominant complaints at onset were a breast mass with local pain and inflammation in four $(66.7 \%)$ patients and a breast mass with pain and without signs of local skin inflammation in two $(33.3 \%)$ patients. Diagnosis was made by using core biopsy in two (33.3\%) patients and by an incisional biopsy in four $(66.7 \%)$ patients. Abscess drainage was performed in four $(66.7 \%)$ patients, and these patients had fistulation to the skin. All patients had used antibiotics. While ultrasonography (USG) was used in all patients, both USG and magnetic resonance imaging were employed in two patients.

Five $(83.3 \%)$ patients achieved a complete response, and one patient (16.7\%) achieved a partial response after the first course of the treatment. A second course of treatment was given to the patient with
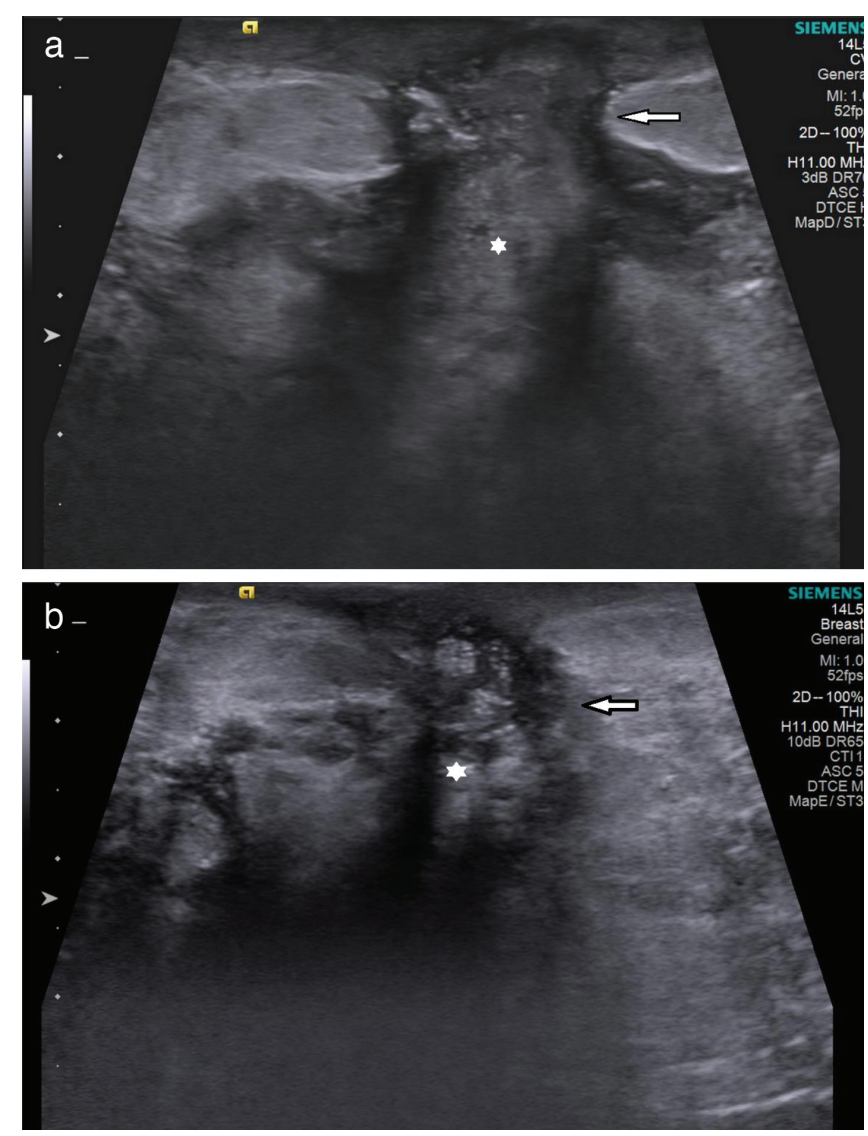

Figure 1. Fistula tract (white arrow) and abscess associated with fistula (white star) before treatment (a), significant regression in the fistula tract (white arrow) and abscess (white star) after treatment (b). (B-mode USG)

USG: Ultrasonography 
partial response. Complete response was achieved by all patients at the end of the second course of treatment (Figures $2 \mathrm{a}$ and $\mathrm{b}$ ). One patient had a recurrence during follow-up at 4 months after delivery, and a third course was administered to this patient. The mean followup time was 19.5 months. Neither topical nor systemic side effects of corticosteroids were observed in any patient. Patients and their clinical characteristics are shown in the accompanying table (Table 1).

\section{Discussion and Conclusion}

IGM is characterized by non-caseating chronic granulomatous lobules in which microorganisms are absent. Causes include local trauma, extravasated secretions, underlying auto-immune process, or damage to the ductal epithelium of an unknown infective etiology. This process is thought to induce a localized immune response that may elicit a local granulomatous response. $(4,7)$ Uncertainty over the exact cause

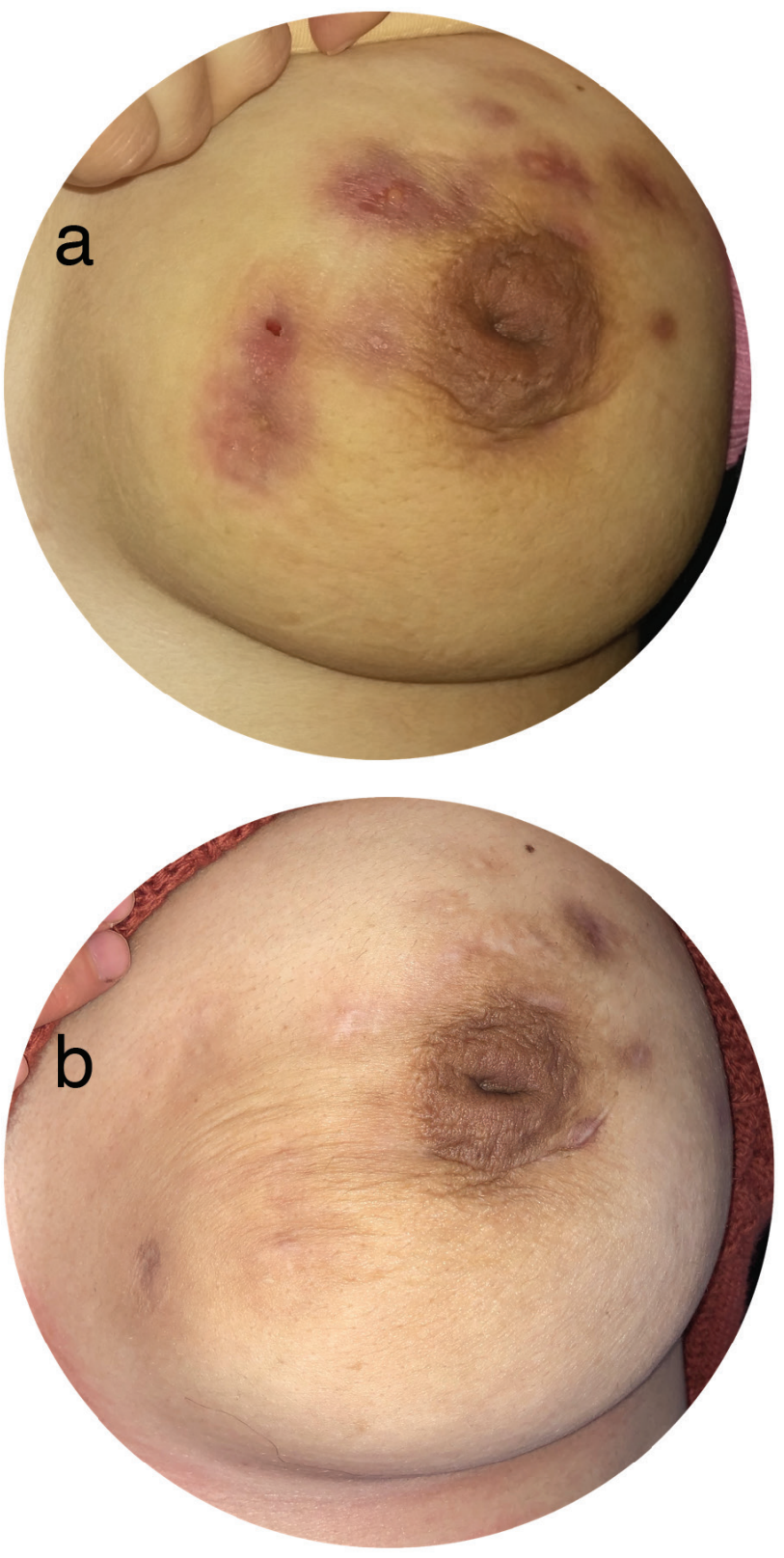

Figure 2. Widespread inflammation in the upper and inner quadrants of the left breast and multiple fistula orifices on the skin, before treatment (a), after treatment (b) of IGM leads to a lack of clear consensus in its treatment. Research on the treatment of IGM indicates that corticosteroids and surgery are predominantly used in IGM treatment (8-10). Since the literature indicates that the number of pregnant women with IGM is low, there is little information about the effectiveness of IGM treatment in pregnant women. A few case reports described pregnant women with IGM who received oral prednisone $(4,11,12)$. By contrast, the present study focused on the assessment of the effect of topical corticosteroid on IGM in pregnant women.

Recurrence rates would likely be higher after limited excision; by contrast, wide local excision may lead to poor cosmetic results. Therefore, surgery should be reserved for recurrent disease or disease unresponsive to medical therapy (4). Many medical treatment modalities cannot be used during pregnancy, with the result that surgery is used more frequently in these patients (11-14). Among the six patients, abscess drainage and incisional biopsy were performed in four patients. In these patients, postoperative wound healing required considerable time; in three patients with abscess drainage, recovery took approximately 4 weeks.

Corticosteroids cause significant regression in breast mass and skin lesions, but high-dose and long-term use of corticosteroids has side effects. Topical corticosteroids are used because of their anti-inflammatory, vasoconstrictor, antiproliferative, and immunosuppressive properties in treating various skin diseases. In addition, corticosteroid has been used in pregnant women for rheumatoid arthritis, lupus, antiphospholipid syndrome, and risk of miscarriage $(15,16)$. In our study, which compared the results of treatment with topical and systemic steroids as described in the literature, patients treated with topical steroids had a shorter duration of treatment, had fewer steroid-related side effects, had less need for surgery, and had lower recurrence rates than systemic steroids (1).

Table 1. Descriptive properties

\begin{tabular}{|c|c|c|}
\hline & & n (\%/range) \\
\hline Mean age & & $26(23-33)$ \\
\hline Mean duration of compla & ints (month) & $4.3(2-6)$ \\
\hline Mean number of children & & $2(2-5)$ \\
\hline Mean breast-feeding tim & (month) & $41(24-72)$ \\
\hline & Left & $3(50)$ \\
\hline Localization & Right & $2(33.4)$ \\
\hline & Bilateral & $1(16.6)$ \\
\hline & $\begin{array}{l}\text { Breast mass with } \\
\text { local pain and } \\
\text { inflammation }\end{array}$ & $4(66.7)$ \\
\hline Main complaint at onset & $\begin{array}{l}\text { Breast mass and } \\
\text { pain without } \\
\text { inflammation }\end{array}$ & $2(33.3)$ \\
\hline Diaanosis & Tru-cut & $2(33.3)$ \\
\hline 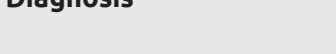 & Incisional & $4(66.7)$ \\
\hline Treatment resnonse & Complete & $5(83.3)$ \\
\hline 7е & Partial & $1(16.7)$ \\
\hline Mean follow-up time (mon & & $19.5(14-28)$ \\
\hline
\end{tabular}


Use of topical steroid in the treatment of IGM was also assessed in other studies $(8,11,17-19)$. Cetin et al. (8) showed no significant difference between the efficacy rates of topical and systemic steroid treatment. Altintoprak et al. (17) showed that clinical improvement occurred within an average of 8.2 weeks in IGM treated with topical steroids, with a long-term success rate of $>90 \%$ during a mean followup duration of 37.2 months. No topical steroid-related side effects were reported during the treatment period.

Goldberg et al. (11) reported that pregnant women with IGM did not respond to 5-week antibiotic treatment and low-dose oral corticosteroid treatment, and the breast mass remained stable until delivery. Breast lesions improved within 2 months with $60 \mathrm{mg}$ corticosteroid prescribed daily postpartum.

Goulabchand et al. (20) treated a 32-week pregnant woman with IGM with antibiotics, but the symptoms persisted until delivery. After 1 month of breastfeeding, breast inflammation aggravated. Breastfeeding was stopped, and corticosteroid treatment was started. After a few months, complete resolution of wounds was achieved, and corticosteroids were tapered and ultimately stopped. Complete remission was observed during early follow-up. Boukaidi et al. (21) used a number of treatment modalities in treating pregnant patients with IGM, including antibiotherapy with nonsteroidal anti-inflammatory drugs, progestogens, vein tonicity drugs, and bromocriptine. Notwithstanding, they could not cure the disease, short of terminating the pregnancy, and used corticosteroids.

Garcia-Rodiguez et al. (2) reported the case of a patient in her $11^{\text {th }}$ week of pregnancy. At the time, the patient's breast mass measured 3 $\mathrm{cm}$, but measured $12 \mathrm{~cm}$ after 10 days. As the patient's pain became intolerable, she was hospitalized twice over 1.5 months. The patient also consulted with other clinics, among them internal medicine, emergency medicine, general surgery, gynecology, and infectious diseases. The patient was treated sequentially with clindamycin, cefazolin, vancomycin, piperacillin-tazobactam combination, ceftriaxone, and metronidazole. She showed no improvement, and narcotics were required to control her pain. After the first dose of vancomycin, the patient developed symptoms of the "red man" syndrome. She also had an allergic reaction to clindamycin. Further bacterial cultures, fungal cultures, and tuberculosis cultures and stains proved negative. After the diagnosis of IGM, the woman was treated with $20 \mathrm{mg}$ prednisone four times daily, and symptoms were improved subsequently. Because of worsening symptoms, treatment options were re-evaluated by a multidisciplinary team, and mastectomy was recommended 1 month after delivery, but the patient did not accept the decision.

All the aforementioned studies show clearly the challenge of treating pregnant patients with IGM. In our study, we used intralesional injection of $40 \mathrm{mg}$ methylprednisolone and topical administration of $0.125 \%$ prednisolone pomade. Within 2 months after treatment, complete remission was apparent in all our patients. Treating patients with topical corticosteroid has the following advantages: stress reduction, increased patient compliance, and a reduction in the cost of treatment. No side effects related to steroids were observed in either the mother or baby. Patients showed no problems with breastfeeding after birth. Moreover, no negative cosmetic results, which are common in repeated surgeries, were observed. Our patients' compliance and satisfaction with this topical steroid treatment, in which only two
In conclusion, the treatment discussed should be considered the first course of action to the treatment of IGM in pregnant women due to the relative ease in delivering a steroid injection and applying topical steroid pomade, more positive response seen in a relatively short time, high patient compliance with treatment, and lower rates of side effects and recurrence.

Ethics Committee Approval: This study was approved by the Local Ethics Committee of Yüzüncü Yll University, Van, Turkey, with the registration number of 2020/04-33.

Informed Consent: Informed consent was received from each patient.

Peer-review: Externally and internally peer-reviewed.

Conflict of Interest: The authors declare that there are no potential conflicts of interest with respect to the research, authorship, and/or publication of this article.

Financial Disclosure: The authors received no financial support for the research, authorship, and/or publication of this article.

\section{Authorship Contributions}

Surgical and Medical Practices: O.T.; Conception: O.T., N.T.; Design: O.T., N.T.; Data Collection and/or Processing: O.T., N.T.; Analysis and/or Interpretation: O.T., N.T.; Literature Search: O.T.; Writing: O.T., N.T.

\section{References}

1. Toktas O, Konca C, Trabulus DC. Soyder A, Koksal H, Karanlik H. et al. A novel first-line treatment alternative for noncomplicated idiopathic granulomatous mastitis: combined intralesional steroid injection with topical steroid administration. Breast Care 2021; 16: 181-187. [Crossref]

2. Garcia-Rodiguez JA, Pattullo A. Idiopathic granulomatous mastitis: a mimicking disease in a pregnant woman: a case report. BMC Research Notes 2013; 6: 95. (PMID: 23497626) [Crossref]

3. Ocal K, Dag A, Turkmenoglu O, Kara T, Seyit H, Konca K. Granulomatous mastitis: clinical, pathological features, and management. Breast J 2010; 16: 176-182. (PMID: 20030652) [Crossref]

4. Gunduz Y, Altintoprak F, Ayhan TL, Kivilcim T, Celebi F. Effect of topical steroid treatment on idiopathic granulomatous mastitis: clinical and radiologic evaluation. Breast J 2014; 20: 586-591. (PMID: 25228089) [Crossref]

5. Alper F, Karadeniz E, Guven F, Cenkaya BY, Ozden K, Akcay MN. The evaluation of the efficacy of local steroid administration in idiopathic granulomatous mastitis: The preliminary results. Breast J 2020; 26: 309311. (PMID: 31495032) [Crossref]

6. Mizrakli T, Velidedeoglu M, Yemisen M, Mete B, Kilic F, Yilmaz H. et al. Corticosteroid treatment in the management of idiopathic granulomatous mastitis to avoid unnecessary surgery. Surg Today 2015; 45: 457-465. (PMID: 24993812) [Crossref]

7. Bani-Hani KE, Yaghan RJ, Matalka I, Shatnawi NJ. Idiopathic granulomatous mastitis: time to avoid unnecessary mastectomies. Breast J 2004; 10: 318-322. (PMID: 15239790) [Crossref]

8. Cetin K, Sikar HE, Goret NE, Rona G, Barisik ON, Kucuk HF. et al. Comparison of topical, systemic, and combined therapy with steroids on idiopathic granulomatous mastitis: a prospective randomized study. World J Surg 2019; 43: 2865-2873. (PMID: 31297582) [Crossref]

9. Barreto DS, Sedgwick EL, Nagi CS, Benveniste AP. Granulomatous mastitis: etiology, imaging, pathology, treatment, and clinical findings. Breast Cancer Res Treat 2018; 171: 527-534. (PMID: 29971624) [Crossref] 
10. Lei X, Chen K, Zhu L, Song E, Su F, Li S. Treatment for idiopathic granulomatous mastitis: systemic review and meta-analysis. Breastfeed Med 2017; 12: 415-421. (PMID: 28731822) [Crossref]

11. Goldberg J, Baute L, Storey L, Park P. Granulomatous mastitis in pregnancy. Obstet Gynecol 2000; 96: 813-815. (PMID: 11094217) [Crossref]

12. Destek S, Gul OV. Idiopathic granulomatous mastitis: a disease mimics breast cancer appearing in pregnancy. Gen Surg: Open Access 2018; 1: 7-10. [Crossref]

13. Yabanoglu H, Colakoglu T, Belli S, Aytac HO, Bolat FA, Pourbager A. et al. A comparative study of conservative versus surgical treatment protocols for 77 patients with idiopathic granulomatous mastitis. Breast J 2015; 21: 363-369. (PMID: 25858348) [Crossref]

14. Skandarajah A, Marley L. Idiopathic granulomatous mastitis: a medical or surgical disease of the breast? ANZ J Surg 2015; 85: 979-982. (PMID: 25424519) [Crossref]

15. Palmsten K, Rolland M, Hebert MF, Clowse MEB, Schatz M, Xu R. et al. Patterns of prednisone use during pregnancy in women with rheumatoid arthritis: Daily and cumulative dose. Pharmacoepidemiol Drug Saf 2018; 27: 430-438. (PMID: 29488292) [Crossref]

16. Empson MB, Lassere M, Craig JC, Scott JR. Prevention of recurrent miscarriage for women with antiphospholipid antibody or lupus anticoagulant. Cochrane Database Syst Rev 2005; 2005: CD002859. doi: 10.1002/14651858.CD002859.pub2 (PMID: 15846641) [Crossref]

17. Altintoprak F, Kivilcim T, Yalkin O, Uzunoglu Y, Kahyaoglu Z, Dilek ON. Topical steroids are effective in the treatment of idiopathic granulomatous Mastitis. World J Surg 2015; 39: 2718-2723. (PMID: 26148520) [Crossref]

18. Altintoprak, F, Kivilcim T, Ozkan OV. Aetiology of idiopathic granulomatous mastitis. World J Clin Cases 2014; 2: 852-858. (PMID: 25516860) [Crossref]

19. Karanlik H, Ozgur I, Simsek S, Fathalizadeh A, Tukenmez M, Sahin D. et al. Can steroids plus surgery become a first-line treatment of idiopathic granulomatous mastitis? Breast Care (Basel) 2014; 9: 338-342. (PMID: 25759614) [Crossref]

20. Goulabchand R, Perrochia H, Aubert-Bringer E, Do Trinh P, Guilpain P. Idiopathic granulomatous mastitis responding to oral prednisone. Breast J 2020; 26: 281-283. (PMID: 31686425) [Crossref]

21. Boukaidi ML, Ghazli M, Bennani O, Hermas S, Soummani A, Bouhya S. et al. [Granulomatous recurrent mastitis during pregnancy.] Gynecol Obstet Biol Reprod (Paris) 2000; 29: 102-104. (PMID: 10675840) [Crossref] 